\title{
Ethical issues that confront nurses in private hospitals in the Western Cape Metropolitan area
}

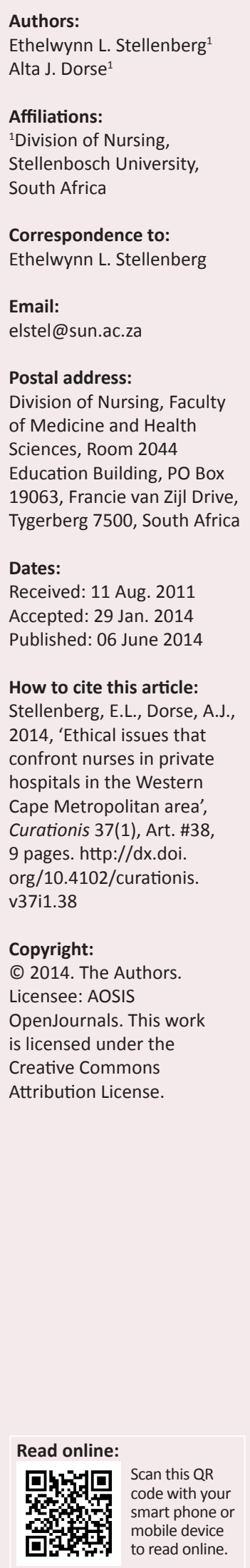

Background: Nurses are faced daily with a variety of ethical issues which could be as a result of budget cuts, target setting, the shortage of nurses and expertise.

Objectives: The objectives of the study were to identify ethical issues related to patient care, to describe ethical issues related to patient diversity, rights and human dignity. To describe ethical issues related to caring in nursing and to the workplace environment.

Method: A quantitative explorative descriptive research design was applied. A stratified sample of $(n=142 / 5 \%)$ was drawn from all nurses and caregivers $(N=2990)$ working in a selected group of eight private hospitals. Self-administered questionnaires were used to collect the data. Statistical tests were applied to determine statistical relationships between variables.

Results: Results included (95\%) of respondents provided safe and committed care to their patients, (99\%) loved to care for their patients and (93\%) believed in the Nurses' Pledge of Service. Fifty percent $(50 \%)$ of the respondents indicated verbal abuse from patients and only (59\%) experienced openness and transparency in the work environment. Analysis further identified that the caregivers did not respect the noble tradition of the profession and experienced the most verbal abuse.

Conclusion: This study has identified ethical issues which may give rise to conflict within the workplace environment if not adequately addressed by management. The study further showed that the use of caregivers not regulated in nursing practice may pose as a threat to the safety of the patient.

\section{Introduction}

Globally nurses are experiencing ethical issues in health and nursing due to a variety of problems which include austerity measures, cutting costs and driven by having to set targets. These measures are in most cases implemented at the cost of patient care, their fundamental basic human rights of respect and human dignity. The inquiry of the Stafford Scandal in England (McFarnon 2013) established that health care was driven by cost-cutting and a target-centred atmosphere which, as reported by Andrew Lansley the Health Secretary, resulted in 'a culture of fear [that] existed in which staff did not feel able to report concerns' and 'a culture of secrecy' (McFarnon 2013:1).

Musa, Harun-Or-Rashid \& Sakamoto (2011:1-7) found in their study conducted in Malaysia that nursing managers are faced with ethical issues on a daily basis, with more than $(59.7 \%)$ experiencing stress when dealing with these ethical issues, the most critical being 'working with unethical or incompetent colleague[s].' It seems to be no different in South Africa today, as nurses are also confronted with ethical issues within the clinical environment which creates daily challenges for decision making. This could be illustrated by the appointment of caregivers, who are not regulated and who have no formal training to be able to participate in nursing activities in hospitals for acute patients as observed by the researchers.

Environments that are conducive to safe, competent and ethical care of patients are dependent on seven primary values that are central to ethical nursing, these include nurses (1) valuing the health and well-being of patients or persons, (2) respecting and promoting the autonomy of patients or clients and helping them to select their choice of health services, (3) valuing and advocating the dignity and self-respect of human beings (4) maintaining confidentiality and safeguarding the trust of clients or patients, (5) applying and promoting the principles of equity and fairness to ensure unbiased treatment, (6) being accountable and consistent in maintaining professional responsibilities and standards of practice and (7) advocating practice environments that promote organisational and human support, to provide safe, competent and ethical nursing care (Burkhardt \& Nathaniel 2002:408-413).

The purpose of this paper is therefore to describe the ethical issues nurses were confronted with as identified in a research study at a selected group of private hospitals. The aim of the research 
was to explore and describe ethical issues that confront nurses in selected private hospitals in the Western Cape Metropolitan area and investigate ethical issues of nursing practice, related to direct patient care, patient diversity, patient rights and human dignity, caring in nursing and workplace environment. It became essential to determine whether nurses were still functioning within the ethical framework of the profession despite the adverse effects of various factors such as the shortage of nurses, the burden of disease and the deficit in professional skills as described by Wildschut \& Mqolozana (2008:2).

\section{Problem statement}

The research study was undertaken as it appeared to the researchers that the fundamental ethical principles in nursing practice in South Africa have diminished over time. Nurses are often forced by circumstances to use measures which compromise patient care. Consequently, this may result in the infringement of a patient's rights and human dignity and could result in litigation against a health institution. It was therefore imperative that a scientific investigation be undertaken to determine the ethical issues in nursing practice which confront the nurses within a selected group of private hospitals.

\section{Research Objectives}

The objectives of the study were to:

- Identify ethical issues related to patient care.

- Describe ethical issues related to patient diversity.

- Describe ethical issues related to the patient rights and human dignity.

- Describe ethical issues related to caring in nursing.

- Describe ethical issues related to the works place environment.

\section{Definition of key concepts}

- Caregiver: For the purpose of this study the person is a non-nurse without any training in nursing, who is not legally regulated but practices elementary nursing.

- Ethics: May be defined as an expected standard and behaviour of a group as described in the group's code of professional conduct (Creasia \& Parker 2001:273). Ethics is fundamentally '... concerned with the meaning of words such as right, wrong, good, bad, ought and duty.' Individuals or people collectively may decide what is right or wrong and acceptable to such an individual or group as found in the nursing profession (Pera \& Van Tonder 2011:5).

- Nurse refers to a person registered in a category under section 31(1) in order to practise nursing or midwifery in terms of the Nursing Act, South Africa (Act 33 of 2005).

- Nursing Practice is referred to as a comprehensive environment within which a nurse practices, that includes: the code of conduct, ethical code and values, standards for practice, competency framework and scope of practice which provides the framework for nursing practice (South African Nursing Council [SANC], 2012).
- Private hospital is a hospital registered as a private company which is operated for profit (Stedman's Medical Dictionary).

\section{Contribution to the field}

The completion of this study has identified ethical issues which may give rise to conflict within the workplace environment if not adequately addressed by management. The study further showed that the use of caregivers not regulated in nursing practice may pose as a threat to safe patient care due to the non-formal training of a nurse. The core values of caring, commitment and compassion of the profession are emphasised and the wish that nurses continue to value the Nurses' Pledge of Service.

\section{Literature review}

According to the International Council of Nurses (ICN) (2006:1), the nurse has four fundamental responsibilities namely to promote health, prevent illness, to restore health and alleviate suffering. Embedded in the nursing practice is the respect for human rights which includes cultural rights, the right to life and choice, to dignity and be treated with respect. Furthermore, practice of nursing takes place within a complex and dynamic environment with the patient as the first consideration (SANC 2013:2-8). Nursing crosses all boundaries and is '... unrestricted by considerations of age, colour, creed, culture, disability or illness, gender, sexual orientation, nationality, politics, race or social status' (ICN 2006:1).

However, personnel are often challenged by serious decisions that have to be made concerning ethical issues on a day-today basis. The most frequently occurring ethical issues in practice include protecting the patient's rights and human dignity, respecting or not respecting informed consent to treatment and providing care with a possible risk to the nurse's health (Mahoney \& Field 2002:9-10).

Whilst nursing practice is challenged by various ethical issues, registered nurses, staff nurses and nursing management are additionally faced with three types of ethical conflicts, namely the professional role, organisational and interpersonal conflict (Riley 2002:1-2). Nurses have the power to make a difference by acting according to the underlying principles that govern their practice. Ethics is the foundation of committed service to humankind and every professional nursing practitioner takes pride in his or her profession (Pera \& Van Tonder 1996:21). According to Pera and Van Tonder (2011:5) ethics is defined as 'concerned with the meaning of words such as right, wrong, good, bad, ought and duty.' Individuals or collectively people may decide what is right or wrong and acceptable to such an individual or group as found in the nursing profession. The noble tradition of the nursing profession has always been the foundation of nursing. Poor ethics and reduced professional standards damage the image of this noble profession (Muller 2009:3).

The legislative framework within which nurses and midwives practise with regard to safe nursing care is stipulated in 
the Nursing Act 2005 (South Africa Act No. 33 of 2005). However, the ethical considerations that determine the relationship of the nurse with both hospital and patient are less clearly defined. Yet, the obligation continues to rest on individual nurses and the nursing profession collectively to achieve quality and safe patient care within a legal and ethical framework. This obligation places the nurse in a vulnerable and precarious position, resulting in exploitation and abuse of the receivers of the care. The danger exists that in such situations the practice of ethics becomes secondary. Ethical and morally acceptable behaviour can easily be deharmonised by burn-out and poor working conditions. Substantiated by Rafii, Oskouie \& Nikravesh (2004:1-3) who mention that a heavy work load has become one of the reasons why many nurses leave the nursing profession or suffer from fatigue and burn-out.

Ethics could be defined as the expected standard and behaviour of a group as described in the group's code of professional conduct (Creasia \& Parker 2001:273). Searle, Human \& Mogotlane (2009:267) state that ethical codes for professions set the parameters of the responsibilities that nurses have with regard to their patients. Professional ethics are moral dimensions of attitude and behaviour based on values, judgement, responsibility and accountability that nurse practitioners take into account when weighing up the consequences of their professional actions.

The ethical foundation of the nursing profession in South Africa is vested in the Nurses' Pledge of Service. This pledge was derived from the Nightingale Pledge and has been in use since in the institution of nurses' training in South Africa. When taking the pledge, the nurse and/or midwife enter into a verbal agreement with the community. The question, however, arises as to whether this pledge reflects the dominant views of nurses in South Africa or not (Muller 1997:14; 2009:3).

Barriers do exist in the workplace which may hamper a compatible ethical environment which creates ethical issues detrimental to decision making. The shortage of nurses has reached crisis proportions as reported in a research study, stating that South Africa has only 222 registered nurses for every 100000 people (Solidarity Research Institute 2009:4-5). In addition to a shortage of nurses, there is also a skills deficit of not only 10250 registered professional nurses but also specific advanced clinical skills, namely 4120 primary health care nurses which makes a total need of 14370 nurses within the nursing profession (Wildschut \& Mqolozana 2008:2). This crisis is further aggravated by the burden of disease. HIV in South Africa increased from an estimated 4.10 million in 2001 to 5.24 million by 2010 (Statistics South Africa 2011:3). Furthermore, the profession is challenged by globalisation, which includes both positive advances in communication and biotechnology but also negative social trends such as poverty which is closely related to health problems (Da Silva 2008:787-790); global changes specifically related to ongoing improvements in technology (Browning, Salsberry \& Reinhardt
2007:1); economic constraints; changing needs of society (Searle 2000:389); and the millennium development goals as recommended by the United Nations (UN) (United Nations Report 2006:3). The overall shortage of nurses and the lack of professional skills result in staff having to work longer hours to provide the required patient care (McIntosh \& Stellenberg 2009:11-20). In an attempt to remedy shortages, the introduction of caregivers not registered with the South African Nursing Council (SANC) into the clinical field to deliver basic care, is currently a grave concern as experienced by the researchers.

Excessive workloads, unsafe working conditions and inadequate supervision can be considered as forms of violence, and are incompatible with good or best clinical practice. Violence in the health work place threatens the delivery of effective patient services and therefore, patient safety. If quality care is to be provided, nursing personnel must be ensured of a safe work environment and respectful treatment (ICN 2007:1).

In South Africa employers should ensure that the Basic Conditions of the Employment Act (South Africa Act 75 of 1997), as amended in 2002 and Labour Relations Act (South Africa Act 66 of 1995) as amended in 2008 are applied to promote health and ethically sound relations in the workplace.

\section{Research question}

The research question: 'What are the ethical issues that confront nurses in selected private hospitals in the Western Cape Metropolitan area?' guided the research.

\section{Research methods and design Design}

A quantitative research design was used to explore and describe ethical issues that confront nurses in selected private hospitals in the Western Cape Metropolitan area.

\section{Sample}

The population consisted of all nurses and caregivers employed in a selected group of eight private hospitals in the Western Cape Metropolitan area, South Africa.

Stratified random sampling was used to draw a sample size of $(n=142 / 5 \%)$. The eight private hospitals formed the specific strata from which $5 \%$ of the population as described was drawn. This ensured that each hospital was adequately represented. A staff list which listed all the caregivers and nurses alphabetically was provided by each hospital. Every 10th name was then selected from the lists provided to participate in the study (Table 1). No specific ward, age or gender was targeted.

All staff who met the criteria had an equal opportunity to be selected. 
TABLE 1: Hospital sample distribution.

\begin{tabular}{llll}
\hline Hospital & Population & $\begin{array}{l}\text { Sample } \\
\text { distribution }\end{array}$ & $\begin{array}{l}\text { Sample } \\
\text { received }\end{array}$ \\
\hline 1. Life Vincent Pallotti & 400 & 20 & 20 \\
2. Life Kingsbury/Claremont & 450 & 15 & 10 \\
3. Vergelegen Medi-Clinic & 300 & 15 & 15 \\
4. Stellenbosch Medi-Clinic & 240 & 12 & 12 \\
5. Cape Town Medi-Clinic & 400 & 20 & 16 \\
6. Panorama Medi-Clinic & 500 & 25 & 23 \\
7. Durbanville Medi-Clinic & 400 & 20 & 14 \\
8. Constantia Medi-Clinic & 300 & 15 & 14 \\
\hline Total & $\mathbf{2 9 9 0}$ & $\mathbf{1 4 2}$ & $\mathbf{1 2 4}$ \\
\hline
\end{tabular}

Source: Dorse 2008:7

\section{Specific criteria}

All nurses and caregivers practising nursing in all disciplines in the selected group of private hospitals were eligible to participate in the study. These hospitals included: Life Vincent Pallotti, Life Kingsbury/Claremont, Vergelegen Medi-Clinic, Stellenbosch Medi-Clinic, Cape Town MediClinic, Panorama Medi-Clinic, Durbanville Medi-Clinic and Constantia Medi-Clinic. Only private hospitals situated in the Cape Metropolitan area, that gave permission to participate in the study, were selected.

\section{Pilot study}

Ten percent $(n=15)$ of the actual sample $(n=142)$ was obtained for a pilot study conducted under circumstances similar to those of the actual study at one of the private hospitals not included in the study. The purpose of the pilot study was to test the feasibility of the methodology, including the instrument for any ambiguity and errors.

\section{Data collection methods}

A self-administered questionnaire with two sections was designed and validated: Section A consisted of biographical data and section B comprised of a Likert scale with questions based on the research objectives. The researcher distributed 142 self-administered questionnaires according to the names identified on the roster of each hospital. The questionnaires in sealed envelopes were left with the respondents to complete and were collected a few days later. Data collection occurred over a period of two months. A response rate of $87 \%$ was achieved.

\section{Data analysis}

The data were analysed by means of the Statistica 7.1, a statistics computer soft ware programme. Data were expressed in frequencies, percentages and tables. The Chi-square, Spearman and Kruskal-Wallis statistical tests were applied to identify associations of statistical significance between variables on a $95 \%$ confidence interval.

\section{Validity and reliability}

Construct, face and criterion validity was ensured through consultation with experts in research methodology, nursing and statistics. A pilot study was conducted to support the reliability and validity further.

\section{Results}

Biographical data: Respondents $(n=124)$ included registered nurses $(48 \%)$, staff nurses $(16 \%)$, auxiliary nurses $(7 \%)$, caregivers (7\%) and nursing management (22\%). The majority of the respondents $(69 \%)$ were in the age group 30-49 years. Most respondents (35\%) had more than 20 years of nursing experience followed by (28\%) between 16 and 20 years of experience.

Data related to ethical issues: Guided by the statistician the data were collapsed to 'agreeing' or 'disagreeing' and 'not applicable' (N/A) responses. The research results are reported in three sections: ethical issues related to the patient, the profession and the hospital as shown in Tables 2-6. Due to the complexity of the results the tables may not reflect all the results.

\section{Section 1: Ethical issues related to the patient}

For the purpose of this discussion the ethical issues relating to the patient are divided into three subsections: direct patient care, diversity, and patient's rights and human dignity.

\section{Subsection 1: Direct patient care}

For a summary please see Table 2 .

Variable 1: You always provide safe and committed care for your patient $(n=124)$. The results showed that the majority ( $n=118 / 95 \%)$ of respondents always provide safe and committed care to their patients.

Variable 2: You love to care for your patient $(n=124)$. Almost all the respondents $(n=120 / 99 \%)$ indicated that they love to care for their patients.

Variable 3: You do not always act as your patient's advocate $(n=124)$. The majority of the respondents $(n=95 / 77 \%)$ disagreed that they do not always act as their patient's advocates, whilst ( $n=24 / 19 \%)$ agreed with the statement. Registered nurses indicated the highest advocacy for patients whilst caregivers the least.

Variable 4: You feel free to discuss your patients' progress with the doctor $(n=124)$. Most respondents $(n=110 / 89 \%)$ indicated that they felt free to discuss their patients' progress with the doctor, whilst $(n=5 / 4 \%)$ disagreed and $(n=9 / 7 \%)$ indicated that the question was not applicable. A statistical significant difference between the years of experience and respondents who felt free to discuss patients' progress with the doctor was identified (Spearman test $p=0.04$ ). The more experienced respondents were more likely to discuss the patient's progress with the doctor.

Variable 5: You experience verbal abuse by the patient $(n=124)$. It is demoralising to note that some of the respondents $(n=62 / 50 \%)$ reported experience of verbal abuse from patients, however others $(n=48 / 39 \%)$ disagreed and the rest ( $n=14 / 11 \%)$ indicated that the question was not applicable. 
Caregivers reported the most verbal abuse. A significant difference was identified between verbal abuse and the area of work (Kruskal-Wallis $p=0.03$ ). Verbal abuse was more likely to occur in critical care units and in the wards.

\section{Subsection 2: Ethical issues related to patient diversity}

For a summary please see Table 3 .

Variables 6: You do not respect all religions $(n=124)$. Results showed that the majority $(n=101 / 81 \%)$ of the respondents disagreed with the statement that they do not respect all religions, although it is a concern that some $(n=20 / 16 \%)$ of the respondents do agree and $(n=3 / 2 \%)$ indicated that the question was not applicable. A statistical difference was identified between the number of years in current position and respect for all religions (Spearman test $p=0.04$ ).

Variables 7: You do not respect all cultures $(n=124)$. Most respondents ( $n=115 / 93 \%)$ indicated that they do respect all cultures. A statistical difference was identified between older respondents and respect for all cultures (Spearman test $p=0.04$ ). The more experienced and older respondents showed more respect towards the various cultures than the less experienced. Only $4(3 \%)$ indicated that the question was not applicable to them, whilst 5 (4\%) do not respect all cultures.

\section{Subsection 3: Ethical issues related to patient's rights and human dignity}

For a summary please see Table 4 .

Variable 8: You always assure patient privacy $(n=124)$. Most respondents $(n=118 / 95 \%)$ indicated that they always respected patient's privacy, but it is a concern that some of the respondents $(n=5 / 4 \%)$ do not always assure patient privacy.

Variable 9: You do not maintain patient confidentiality $(n=124)$. The majority of respondents in clinical practice $(n=102 / 82 \%)$ disagreed with the statement that they do not maintain patient confidentiality; however it is a concern that $(20 / 16 \%)$ indicated that they do agree with the statement.

Variable 10: You do not acknowledge patient autonomy $(n=124)$. Most respondents $(n=104 / 84 \%)$ disagreed that they do not acknowledge patient autonomy but it is a concern that some of the respondents $(n=15 / 12 \%)$ agreed with the statement that they do not acknowledge patient autonomy and the rest $(n=5 / 4 \%)$ indicated as not being applicable.

Variable 11: You are not always honest with the patient $(n=124)$. It is disheartening to note that $32 \%$ of respondents $(n=39)$ agreed that they are not always honest with patients. It is a further concern that $7 \%(n=9)$ of the respondents indicated that the question was not applicable to them. Honesty forms an integral part of the integrity of an individual and more so in a profession in which clinical practice is based on ethical and moral values.

Variable 12: You safeguard the patient from unethical practice $(n=124)$. The majority of respondents $(n=113 / 91 \%)$ indicated that they safeguarded the patient from unethical practice, but $(n=7 / 6 \%)$ disagreed with the statement and ( $n=4 / 3 \%)$ indicated as not being applicable. Registered nurses showed the highest result in safeguarding the patient from unethical practice.

\section{Section 2: Ethical issues related to caring in nursing}

For a summary please see Table 5 .

Variable 13: Nursing is not a caring and compassionate profession $(n=124)$. The majority of respondents $(n=109 / 88 \%)$ disagreed that nursing is not a caring and compassionate profession but ( $n=14 / 11 \%$ ) agreed with the statement.

TABLE 2: Ethical issues related to patient care.

\begin{tabular}{llll}
\hline Variables & Response & $N$ & $\mathbf{\%}$ \\
\hline $\begin{array}{l}\text { Variable 1: You always provide safe and committed care } \\
\text { for your patient: }(n=124)\end{array}$ & Agree & 118 & 95 \\
& Disagree & 6 & 5 \\
& N/A & 0 & 0 \\
Variable 2: You love to care for your patient: $(n=124)$ & Agree & 122 & 99 \\
& Disagree & 2 & 1 \\
& N/A & 0 & 0 \\
Variable 3: You do not always act as your patient's & Agree & 24 & 19 \\
advocate: $(n=124)$ & Disagree & 95 & 77 \\
& N/A & 5 & 4 \\
Variable 4: You feel free to discuss your patients' & Agree & 110 & 89 \\
progress with the doctor: $(n=124)$ & Disagree & 5 & 4 \\
& N/A & 9 & 7 \\
Variable 5: You experience verbal abuse by the patient: & Agree & 62 & 50 \\
$(n=124)$ & Disagree & 48 & 39 \\
& N/A & 14 & 11 \\
\hline
\end{tabular}

Source: Dorse 2008:57-69

TABLE 3: Ethical issues related to patient diversity.

\begin{tabular}{llll}
\hline Variables & Response & $\boldsymbol{N}$ & $\mathbf{\%}$ \\
\hline Variable 6: You do not respect all religions: $(n=124)$ & Agree & 20 & 16 \\
& Disagree & 101 & 81 \\
& N/A & 3 & 2 \\
Variable 7: You do not respect all cultures: $(n=124)$ & Agree & 5 & 4 \\
& Disagree & 115 & 93 \\
& N/A & 4 & 3 \\
\hline
\end{tabular}

Source: Dorse 2008:56-57

TABLE 4: Ethical issues related to patient's rights and human dignity.

\begin{tabular}{llll}
\hline Variables & Response & $\boldsymbol{N}$ & $\mathbf{\%}$ \\
\hline Variable 8: You always assure patient privacy: $(n=124)$ & Agree & 118 & 95 \\
& Disagree & 5 & 4 \\
& N/A & 1 & 1 \\
Variable 9: You do not assure patient confidentiality: & Agree & 20 & 16 \\
$\begin{array}{l}(n=124) \\
\text { Variable 10: You do not acknowledge patient autonomy: }\end{array}$ & Disagree & 102 & 82 \\
& Agree & 15 & 12 \\
& Disagree & 104 & 84 \\
& N/A & 5 & 4 \\
Variable 11: You are not always honest with the patient: & Agree & 39 & 32 \\
$(n=124)$ & Disagree & 76 & 61 \\
& N/A & 9 & 7 \\
Variable 12: You safeguard the patient from unethical & Agree & 113 & 91 \\
practice: $(n=124)$ & Disagree & 7 & 6 \\
& N/A & 4 & 3 \\
\hline
\end{tabular}

Source: Dorse 2008:56-61 
Variable 14: You believe in the Nurses' Pledge of Service $(n=124)$. Results showed that $(n=115 / 93 \%)$ of the respondents believed in the Nurses' Pledge of Service, whilst $(n=6 / 5 \%)$ do not believe in the Nurse's Pledge and $(n=3 / 2 \%)$ indicated as not applicable to them. The caregivers did not share the same belief.

Variable 15: You are not loyal to the profession $(n=124)$. Most respondents $(n=112 / 91 \%)$ disagreed that they are not loyal to the profession, however $(n=8 / 6 \%)$ agreed with the statement and $(n=4 / 3 \%)$ indicated as not being applicable. The analysis showed that caregivers were the least loyal to the profession. This may be attributed to the fact that the caregivers were not trained nurses.

Variable 16: You market the profession positively $(n=124)$. The majority of the respondents $(n=101 / 81 \%)$ agreed that they market the profession positively whilst some of the respondents $(n=17 / 14 \%)$ indicated that the question was not applicable to them and $(n=6 / 5 \%)$ disagreed.

Variable 17: You do not respect the noble tradition of the profession $(n=124)$. Most respondents $(n=113 / 91 \%)$ disagreed with the statement that they do not respect the noble traditions whilst $(n=6 / 5 \%)$ respondents agreed that they do not respect the noble tradition of the profession and $(n=5 / 4 \%)$ indicated that the question was not applicable. Analysis further identified that the caregivers did not respect the noble tradition of the profession. Caregivers are not trained nurses and are possibly not aware of the noble traditions of the nursing profession.

\section{Section 3: Ethical issues related to the workplace environment}

For a summary please see Table 6 .

In the following section ethical issues relating to factors which may create conflict in the workplace environment are reported on.

Variable 18: You are unhappy in your area of work $(n=124)$. Most respondents $(n=94 / 76 \%)$ indicated that they are not unhappy in their area of work. However $(n=14 / 11 \%)$ indicated that they are unhappy in the workplace and $(n=16 / 13 \%)$ indicated that the question was not applicable. Further analysis showed that caregivers and nurses working in the wards mostly were unhappy in their area of work whilst management was the least unhappy.

Variable 19: Your right to privacy is respected $(n=124)$. Only ( $n=90 / 73 \%)$ of the respondents agreed that their right to privacy is respected but many of the respondents disagreed $(n=20 / 16 \%)$ that their rights were respected and $(n=14 / 11 \%)$ indicated that the question was not applicable. However, it was identified that caregivers indicated that their right to privacy was not respected, whilst nursing management indicated that their right to privacy is respected the most.

Variable 20: You are loyal and committed to the hospital where you are employed $(n=124)$. Most respondents $(n=114 / 97 \%)$ agreed that they are loyal and committed to the hospital where they are employed but $(n=5 / 4 \%$ indicated that the question was not applicable.

Variable 21: You feel exploited in your area of work $(n=124)$. Results showed that $(n=55 / 44 \%)$ of the respondents agreed that they felt exploited in their area of work, but $(n=46 / 37 \%)$ disagreed and $(n=19 / 23 \%)$ indicated that the question was not applicable. A statistical difference was identified between overtime and working 10-19 hours overtime per month (Man-Whitney U test $p=0.01$ ). The Spearman test determined that there was a statistical difference between age and overtime worked ( $p=0.05$ ). The age group 40-49 worked more overtime with registered nurses working the most overtime.

Variable 22: There is openness and transparency in the work environment $(n=124)$. Only $(n=73 / 59 \%)$ of respondents agreed

TABLE 5: Ethical issues related to the caring of nursing.

\begin{tabular}{llll}
\hline Variables & Response & $N$ & $\%$ \\
\hline Variable 13: Nursing is not a caring and compassionate & Agree & 14 & 11 \\
profession: $(n=124)$ & Disagree & 109 & 88 \\
& N/A & 1 & 1 \\
Variable 14: You believe in the Nurses' Pledge of service: & Agree & 115 & 93 \\
$(n=124)$ & Disagree & 6 & 5 \\
& N/A & 3 & 2 \\
Variable 15: You are not loyal to the profession: $(n=124)$ & Agree & 8 & 6 \\
& Disagree & 112 & 91 \\
& N/A & 4 & 3 \\
Variable 16: You market the profession positively: & Agree & 101 & 81 \\
$(n=124)$ & Disagree & 6 & 5 \\
& N/A & 17 & 14 \\
Variable 17: You do not respect the noble tradition of & Agree & 6 & 5 \\
the profession: $(n=124)$ & Disagree & 113 & 91 \\
& N/A & 5 & 4 \\
\hline
\end{tabular}

Source: Dorse 2008:61-63

TABLE 6: Ethical issues related to the workplace environment.

\begin{tabular}{llll}
\hline Variables & Response & $\boldsymbol{n}$ & $\mathbf{\%}$ \\
\hline $\begin{array}{l}\text { Variable 18: You are unhappy in your area of work: } \\
(n=124)\end{array}$ & Agree & 14 & 11 \\
& Disagree & 94 & 76 \\
& N/A & 16 & 13 \\
Variable 19: Your right to privacy is respected: $(n=124)$ & Agree & 90 & 73 \\
& Disagree & 20 & 16 \\
& N/A & 14 & 11 \\
Variable 20: You are loyal and committed to the hospital & Agree & 117 & 94 \\
where you are employed: $(n=124)$ & Disagree & 2 & 2 \\
& N/A & 5 & 4 \\
Variable 21: You feel exploited in your area of work: & Agree & 55 & 44 \\
$(n=124)$ & Disagree & 46 & 37 \\
& N/A & 23 & 19 \\
Variable 22: There is openness and transparency in the & Agree & 73 & 59 \\
workplace environment: $(n=124)$ & Disagree & 20 & 16 \\
& N/A & 31 & 25 \\
Variable 23: Your salary does not match your & Agree & 93 & 75 \\
responsibility: $(n=124)$ & Disagree & 20 & 16 \\
& N/A & 11 & 9 \\
Variable 24: You function in an area where there is trust & Agree & 97 & 78 \\
and common purpose: $(n=124)$ & Disagree & 8 & 6 \\
& N/A & 19 & 16 \\
\hline
\end{tabular}

Source: Dorse 2008:64-66 
that there is openness and transparency in the hospital where they worked. It was noted that $(n=31 / 25 \%)$ indicated the question was not applicable and $(n=20 / 16 \%)$ disagreed. Further analysis showed a statistical difference between openness and transparency in the work environment and the position they held (Spearman test $p=0.03$ ). The management was more likely to agree that there is transparency in the hospital.

Variable 23: Your salary does not match your responsibility $(n=124)$. Respondents ( $n=93 / 75 \%$ ) agreed that their salaries do not match their responsibility, especially registered nurses agreed with the statement. However $(n=20 / 16 \%)$ disagreed and ( $n=11 / 9 \%$ ) indicated that the question was not applicable.

Variable 24: You function in an area where there is trust and common purpose $(n=124)$. Most respondents $(n=97 / 78 \%)$ agreed that they function in an area where there is trust and common purpose, but it is a concern that $(n=19 / 16 \%)$ respondents indicated the question was not applicable and ( $n=8 / 6 \%$ ) disagreed. Management mostly agreed that there is trust and common purpose.

\section{Ethical considerations}

Ethical clearance was obtained from the University of Stellenbosch. Permission to conduct the study was obtained from the management of each participating hospital. Informed consent was obtained from the respondents who were also informed that participation in the study was voluntary and that they could withdraw from the study without any victimisation. Signed consents were collected in separate nameless sealed envelopes.

Each questionnaire without any identity was placed in a sealed envelope and treated with confidentiality and anonymity. Data collected are stored in a locked cupboard which only provides access to the researchers and will be destroyed after five years.

\section{Discussion \\ Outline of results}

The study successfully explored the ethical issues that confront nurses in selected private hospitals in the Western Cape Metropolitan area with specific reference to direct patient care, caring in nursing and the workplace environment.

\section{Ethical issues related to the patient}

The research upon which this article is based has shown that $(99 \%)$ of the respondents love to care for the patient; that $(95 \%)$ of the respondents always provided safe and committed care for the patient and always acted as advocates (77\%) for the patient. However, many respondents (50\%) indicated that they experienced verbal abuse from patients with the caregivers indicating the most verbal abuse and
$(11 \%)$ indicating that the question posed was not applicable to them.

\section{Ethical issues related to caring in nursing}

The majority (93\%) of the respondents believed in the Nurse's Pledge of Service. However, nursing practice is guided by both an ethical and a legal framework. The Nurses' Pledge of Service (SANC 2012) provides an ethical guideline for the nursing profession and aspects of this pledge are directly related to the rights of patients which are based on the rights of individuals as described in the Constitution of South Africa (South Africa Act 108 of 1996). Despite the fact that the majority of the respondents (91\%) indicated that they safeguarded the patients from unethical practice this was not true of all respondents. Adverse responses of the minority of the respondents are unacceptable. The caring and compassion of the majority of respondents were marred by the minority who do not practice according to the Nurses' Pledge of Service or have no respect for the noble tradition of nursing. If only a few respondents disregarded the Nurses' Pledge of Service severe repercussions for nursing practice may result.

Ethical issues related to patient's rights and human dignity The respect for the basic human rights of patients should be no less than $100 \%$. Society expects that the nursing profession respect and honour the moral values of society. These values, described as the basic human rights of an individual are a legal requirement and not only an ethical one. The study showed that $(12 \%)$ of the respondents do not acknowledge a patient's autonomy with $4 \%$ indicating that the question posed was not applicable to them. The rights of patients were aggravated further by some respondents who did not acknowledge the patient's confidentiality (16\%), privacy (4\%) and honesty (32\%).

\section{Ethical issues related to patient diversity}

Only (93\%) respect all cultures and (81\%) all religions.

\section{Ethical issues related to the workplace environment}

Whilst ethical issues related to the patient and the caring ethos of nursing are of utmost importance in nursing practice so too are the basic conditions of service and labour relationships which compliment the nursing practice. Problems in the workplace environment may give rise to ethical issues in the hospital. The study showed that adverse conditions existed in the workplace environment because not all respondents $(11 \%)$ were happy in their area of work, with (13\%) indicating that the question posed was not applicable to them. The right to privacy was not respected $(16 \%)$ with $(11 \%)$ of the respondents indicating that the question was not applicable to them. In addition (44\%) of the respondents indicated that they were exploited in their work and $(75 \%)$ of the respondents indicated that their salaries did not match their responsibility. Only (59\%) indicated that they experienced openness and transparency in their work environment and only (78\%) indicated that there was trust and common purpose. 


\section{Practical implications}

Many respondents indicated 'not applicable' to various questions which for the purpose of this study was noteworthy, as all aspects related to patient care, caring in nursing and the work environment and are applicable to those practising nursing.

Adversarial conditions, no matter how minor in nursing practice create ethical issues which may lead to violation of the patient's basic rights and human dignity, including the ethos of nursing. Contravening the basic human rights of patients may result in disciplinary action (South Africa Nursing Act 33 of 2005) including litigation (South Africa Act 108 of 1996). The Nurses' Pledge of Service is taken as an oath by registered nurses on entry into the profession. This study showed that those who did not believe in the Nurses' Pledge of Service and who did not respect the noble tradition of the profession were amongst the caregivers.

In conclusion, individual basic human rights and labour rights of all citizens are entrenched in the Constitution of the Republic of South Africa (South Africa Act 108 of 1996). The ethos of nursing forms the foundation of nursing practice and provides an ethical framework within which the nurses practise (SANC 2013:1-8). Respect, trust, recognition and empowerment are the key ingredients to worker satisfaction in a health care organisation, and therefore to the success of its performance management system (Katz \& Green 1997:299).

\section{Limitations of the study}

The public sector and certain private hospital groups were not included in this study. In addition, agency nurses were not included, yet they form a critical human resource for private hospitals. According to a Life Healthcare presentation (2007: n.p.) approximately $30 \%$ to $40 \%$ of agency nurses were being used in private hospitals. Therefore the findings of this study could not be generalised to apply to other hospitals.

\section{Recommendations}

It is therefore recommended that employers address shortcomings in the workplace environment to minimise ethical issues which may arise that threaten nursing practice. Trust, integrity, respect and the recognition of the worth of each employee in the workplace will ensure a nonthreatening, safe and secure environment for patient care.

Caregivers who practise nursing are contravening the Nursing Act (South Africa Act 33 of 2005) as they are not regulated. Nursing practice is regulated to protect the patient and the public at large. Formal training aligned to the Nursing Act (South Africa Act 33 of 2005) should be given to caregivers and registration should be considered. Their current use may place the patient's safety at risk, especially in hospitals managing acute patients. This study has shown gaps in the knowledge of especially caregivers who are not trained nurses practising nursing.
Continuous in-service training and in-service training related to ethical issues in nursing, the caring ethos, the basic human rights of individuals and the patient's rights charter (Department of Health 1999:1-3) should be given to all nurses at regular intervals. The critical analysis of case studies or patient scenarios relating to nursing practice should form part of in-service training of those practising nursing. Special days of celebration such as International Nurses' Day could be an opportunity to renew the Nurses Pledge of Service by all nurses.

\section{Conclusion}

The integrity and honour of the profession lies within the power of the individuals who make up the profession. Factors adversely influencing the profession should be addressed vehemently to enable the profession to maintain the tradition of the 'noble' profession and the caring ethos of the profession as shown in the results of the research upon which this paper is based.

\section{Acknowledgements}

The authors acknowledge the private hospitals and participants who willingly agreed to participate in the research being conducted and Illona Meyer who assisted with the language editing.

\section{Competing interest}

The authors declare that they have no financial or personal relationship(s) which may have inappropriately influenced them in writing this article.

\section{Authors' contributions}

E.L.S. (Stellenbosch University) supervised the research and wrote the article for publication. A.J.D. (Stellenbosch University) conducted the research.

\section{References}

Browning, S., Salsberry, P.J. \& Reinhardt, C., 2007, 'Ethical Issues in nursing practice: Identifications and comparisons across clinical specialty' Focusing on EvidenceBased Practice, 18th International Nursing Research Congress, Sigma Theta Tau International, Baltimore, July 11-14, 2007, pp.1-12.

Burkhardt, A.B. \& Nathaniel, A.K., 2002, Ethics \& issues in contemporary nursing, 2nd edn., Delmar,New York.

Creasia, J.L. \& Parker, B., 2001, Conceptual foundations: The bridge to professional nursing practice, 3rd edn., Mosby, St Louis.

Department of Health, 1999, National Patients' Rights Charter, Government Printer, Pretoria.

Da Silva, A.L., 2008, 'Nursing in the era of globalisation: Challenges for the 21st Century' Review Latino-am Enfermagem, julho-agosto 16(4),787-90, viewed 16 September 2011, from http://www.eerp.usp.br/rlae Comunicações Breves/Relato de Casos

Dorse, J.L., 2008, 'Legal and ethical aspects of nursing practice in selected private hospitals in the Western Cape Metropolitan area', MCUR Thesis, Division of Nursing, Stellenbosch University.

International Council of Nurses, 2007, Framework guidelines for addressing workplace violence in the health sector, The Training Manual, viewed 14 September 2007 from http://www.icn.ch

International Council of Nurses, 2006, The ICN Code of Ethics for Nurses, viewed 21 July 2012, from http://www.icn.ch/images/stories/documents/about/icncode english.pdf

Katz, J.M. \& Green, E., 1997, Managing quality: A guide to system wide performance management in health care, Mosby, St Louis.

Life Healthcare Presentation, 2007, Nursing model restructuring - Consultation process, Life Healthcare Conference, Cape Town, May. 
Mahoney, M. \& Field, L., 2002, 'Research Report: Ethics \& Human Rights Issues in Nursing Practice: A survey of Massachusetts Registered Nurses', Nursing Ethics Network, University College of Nursing, Boston/Brigham \& Women's Hospital, Boston.

McFarnon E., 2013, 'Stafford Hospital deaths scandal findings due today', viewed 6 February 2013, from http://www.thisisstaffordshire.co.uk/Stafford-Hospitaldeaths-scandal-findings-today/story-18068645-detail/story.html\#ixzz2K7J1CFuk

McIntosh, J. \& Stellenberg, E.L., 2009, 'Investigation into a staffing strategy based on voluntary increase in working hours in a hospital in Kwa-Zulu Natal' Curationis, June 32(2):11-20.

Muller, M., 1997, Nursing dynamics, 3rd edn., Heinemann, Johannesburg.

Muller, M., 2009, Nursing dynamics, 4th edn., Heinemann, Johannesburg.

Musa, M.B., Harun-Or-Rashid, M.D. \& Sakamoto, J., 2011, 'Nurse managers' experience with ethical issues in six government hospitals in Malaysia: A crosssectional study', BMC Medical Ethics 12(23), viewed 5 February 2013, from http://www. biomedcentral.com/1472-6939/12/23

Pera, S.A. \& Van Tonder, S. 1996, Ethics in nursing practice, 2nd edn., Juta \& Co., Cape Town.

Pera, S.A. \& Van Tonder, S., 2011, Ethics in Healthcare, 3rd edn., Juta \& Co Ltd., Cape Town.

Rafii, F., Oskouie, F. \& Nikravesh, M., 2004, 'Factors involved in nurses' responses to burnout: a grounded theory study', BMC Nursing 3:6, viewed 17 March 2010, from http://www.biomedcentral.com/1472-6955/3/6, http://dx.doi.org/10.1186/1472 6955-3-6

Republic South Africa, 1996, Constitution of the Republic of South Africa Act (Act 108 of 1996), Government Printer, Pretoria.

Republic of South Africa 2002, Basic Conditions of Employment Act (Act 75 of 1997, as amended), Government Printer, Pretoria.
Republic of South Africa, 2005, Nursing Act (Act 33 of 2005), Government Printer, Pretoria. Republic South Africa, 2008, Labour Relations Act (Act 66 of 1995, as amended), Government Printer, Pretoria.

Riley, J.M., 2002, 'Research Report: Nurse Executive's Response to ethical conflict and choice in the workplace', Department of Nursing Emmanuel College, Nursing Ethics Network, Boston.

Searle, C., 2000, Professional Practice: A Southern African Nursing Perspective, 4th edn. Heinemann, Pinetown.

Searle, C., Human, S. \& Mogotlane, S.M., 2009, Professional practice. A Southern African Nursing Perspective, 5th edn., Heinemann, Sandton.

Solidarity Research Institute, 2009, 'Report Nurse Shortage in South Africa. Nurse/ Patient Ratios May', viewed 9 March 2009, from http:// www.solidarity.co.za

South African Nursing Council (SANC), 2012, 'Nurses' Pledge of Service' (Under the provisions of the Nursing Act, 2005).

South African Nursing Council, 2013, Code of ethics for nursing practitioners in South Africa, Government Printer, Pretoria.

Statistics South Africa, 2011, Statistical release Mid-Year Population Estimates 2010, Government Printer, Pretoria.

Stedman's Online Medical Dictionary, viewed August 2012, from http://www.stedmans. com

United Nations, 2006, 'Report of Millennium Development Goals', United Nations Department of Economic and Social Affairs, DESA, New York.

Wildschut, A. \& Mqolozana, T., 2008, ' Shortage of Nurses in South Africa: Relative or Absolute?', Case Study Report, forming part of the Human Sciences Research Council Study: A multiple source identification and verification scarce and critical skills in the South African labour market', Commissioned by the Department of Labour, Government Printer, Pretoria. 